\title{
Case Report: Managing a COVID-19 Nursing Home Outbreak a Physician's Perspective
}

By Dr. Jim Shalom

Abstract- I am the medical director and sole physician at a 35-bed nursing home in Western Galilee in Israel. At the end of December 2020, the nursing home experienced a COVID outbreak in which nineteen residents were infected. Four of them were symptomatic, and two died. Ten staff members, including our administrator, likewise caught the disease, and five of them were symptomatic. All recovered. The first part of this paper will examine why, despite all our precautions, the outbreak occurred when it did, and why it spread extensively within the home. The second part will describe how the nursing home functioned after the outbreak, until its residents recovered.

GJMR-K Classification: NLMC Code: WB 105

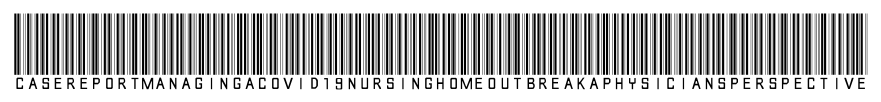

Strictly as per the compliance and regulations of:

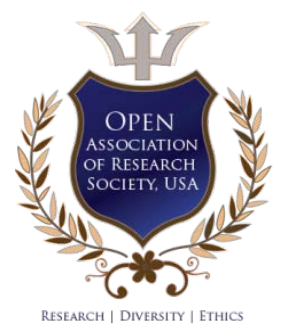

(c) 2021. Dr. Jim Shalom. This is a research/review paper, distributed under the terms of the Creative Commons AttributionNoncommercial 3.0 Unported License http://creativecommons.org/licenses/by-nc/3.0/), permitting all non-commercial use, distribution, and reproduction in any medium, provided the original work is properly cited. 


\title{
Case Report: Managing a COVID-19 Nursing Home Outbreak a Physician's Perspective
}

\author{
Dr. Jim Shalom
}

\begin{abstract}
I am the medical director and sole physician at a 35bed nursing home in Western Galilee in Israel. At the end of December 2020, the nursing home experienced a COVID outbreak in which nineteen residents were infected. Four of them were symptomatic, and two died. Ten staff members, including our administrator, likewise caught the disease, and five of them were symptomatic. All recovered. The first part of this paper will examine why, despite all our precautions, the outbreak occurred when it did, and why it spread extensively within the home. The second part will describe how the nursing home functioned after the outbreak, until its residents recovered.
\end{abstract}

\section{INTRODUCTION}

1 $\mathrm{n}$ recent months, numerous published papers by experts in various fields, including economists, ${ }^{1}$ geographers, ${ }^{2}$ and physicians, ${ }^{3}$ have analyzed nursing home outbreaks of COVID-19. To the best of my knowledge, none of these descriptions is based on first-hand experience. This paper will describe and analyze a COVID-19 outbreak in the nursing home where I work. ${ }^{4}$ I am the medical director and sole physician at a 35-bed nursing home in Western Galilee, in Israel. Until December 2020, none of our residents had been infected with COVID-19. At the end of December, however, one of our nurses was found to be COVID positive. Despite feeling unwell, she had reported for work, as she is not given to complaining and was well aware of our COVID-related staffing issues. She did not inform the nursing home administration of her illness, but that same day, after completing her shift, she went to her physician, who diagnosed her with strep throat and prescribed antibiotics. Since neither she nor he imagined she had COVID, she was not tested. When she still felt unwell four days later, however, she went for a COVID test and received a positive result that evening. She can recall no possible infectious contact. I should add that she had been careful throughout the entire pandemic, and that now, too, she strove to adhere to COVID protocols and denies having touched any of the residents on the day she felt unwell. However, because of her diagnosis, two residents who had had a fever that day were sent to the E.R. for emergency testing, and one was found to be COVID positive. Because she was the only positive contact, we presume that she was the vector that

Author: e-mail: jim.shalom@gmail.com introduced the virus. Despite our best attempts, COVID19 had penetrated our nursing home.

All our residents and workers were group-tested the following day, and when the results came back two days later six additional residents were found to be COVID positive. We were in the throes of an outbreak.

Ministry of health policy dictated that all COVIDpositive residents were to be transferred out. Those who were significantly symptomatic were referred to hospital for admission to COVID wards, while those who were asymptomatic were referred to one of two other regional nursing homes that were equipped with special facilities and designated for COVID residents.

We pursued a policy of routine testing for all workers and residents every three days, under an arrangement conveniently coordinated with the ministry of health, which sends a team to test all our nursing home staff and residents on-site at one go, regardless of which of the four national health funds they happen to belong to. In addition to each person's receiving their own results, all findings are collectively sent to the medical director or administrator. Unfortunately, because of laboratory backlogs from increased nationwide testing, up to 2 days would pass before results were obtained. All told, 19 residents were infected, 2 of whom died of COVID. Ten out of 30 healthcare workers were likewise infected, of whom 5, including our administrator, were symptomatic. None required hospitalization.

There may well be a presumption that any nursing home that experiences a COVID outbreak must have been guilty of negligence. While it is true that some outbreaks were associated with understaffing, inadequate use of protective gear, and poor implementation of COVID protocols, ${ }^{5}$ detailed analysis will show that lax observance of COVID procedures is only one of two possible explanations, and that nursing homes that have strictly followed protective guidelines have also experienced outbreaks. ${ }^{6}$

Let's start with the dos, don'ts, and practical difficulties associated with preventing nursing home outbreaks.

Throughout the pandemic, we strove to take all appropriate precautionary measures. From the outset, we had an available supply of PPE (personal protective equipment), including gloves, masks, gowns, and soaps, and their use was immediately and appropriately implemented. With regard to the CDC guidelines, ${ }^{7}$ the 
only step we failed to take was the designation of a specific person responsible for IPC (infection and protection control). In our case, this task was divided and coordinated between the administrator, the head nurse, and me.

- With regard to minimizing outbreaks caused by infected health workers, the literature stresses the importance of identifying staff members who feel ill and ensuring that they stay home from work. ${ }^{8}$ However, such a change cannot be effectively instituted without a paradigm shift, as sick leave is typically granted only when the state of an employee's physical health inhibits their performance at work. ${ }^{9}$ Taking time off for minor complaints because of the risk of COVID involves a change in thinking on the part of both employer and employee, particularly among disciplined workers with a strong sense of duty. It requires a different and lower threshold for considering missing work, and a new reason for staying away, beyond reduced performance capacity. Then there are the staffing implications: being understaffed has the inadvertent paradoxical consequence of causing staff members to feel obliged to report for work even when slightly unwell. There is no easy solution to this problem. Hiring new help at such a critical time, when peak performance skills are essential from the outset, is not an appealing expedient.

- In addition to adhering to this new absenteeism protocol, we have insisted from the very beginning that health workers take appropriate safety precautions, such as remaining at home as much as possible and being selective about whom they encounter. Sensitivity to the home prevention factor has been part and parcel of our COVID infection control policy.

- In our nursing home, two residents generally share a room. During the pre-pandemic period, this was a practical and effective arrangement. However, during the outbreak, we found that if one resident of a room was COVID positive, the other was often also found to be so when tested 3 days later. This finding is consistent with the literature, in which there is a correlation between increased infection rates and facilities with predominantly double rooms and other space constraints. ${ }^{10}$ Moreover, a nursing home structure is not a "musical chairs" situation in which any desired urgent re-arrangement is implementable.

Despite these limitations, we managed to keep the nursing home free of COVID for over 9 months.

The first question I want to address is why the virus penetrated the nursing home at this particular time?

Despite the presumption that the higher the level of care and infection control, the smaller the likelihood of viral invasion, growing evidence demonstrates that outbreaks have also occurred in nursing homes with five-star ratings. One report concludes: "COVID-19 cases in nursing homes are related to facility location and size and not traditional quality metrics such as star rating and prior infection control citations." 11 Gorges and Konetzka have reached the same conclusion: "The strongest predictor of cases and outbreaks in nursing homes is per capita cases in the county." 12

These findings are consistent with our own experience. During the month of December, the community incidence of COVID-19 was the highest it had ever been, and thus our outbreak coincided directly with elevated COVID-19 rates in the surrounding communities. I should add that during this period there were parallel outbreaks in other nursing homes in Israel, which again correlate with a high COVID-19 community incidence.

The next question is: Once the virus is in the nursing home, why is the spread relentless and usually unpreventable?

The WHO protocols emphasize early detection among health workers and implementation of preventative procedures. ${ }^{13}$ While these are important, I believe that there will be situations such as ours in the future, too, in which adherence to these protocols does not prevent internal viral spread.

There are several reasons for this. Let me posit three:

1. In addition to the common contagious mechanism of person-to-person spread through large droplets, there exists a secondary mechanism that, though less contagious, is not negligible, and which, under certain circumstances, may contribute significantly to spread. In their paper "Beyond Six Feet: A Guideline to Limit Indoor Airborne Transmission of COVID-19," Bazant and Bush describe this secondary mechanism of spread, ${ }^{14}$ which consists of small aerosol droplets that remain suspended for long periods within closed, well-mixed indoor spaces. In a case study of a nursing home environment in which an infected person is present, they conclude that the six-foot rule will fail after 17 minutes, i.e., that there is a significant likelihood of spread from an infected person to an uninfected person within 17 minutes. Furthermore, the authors assert that infected indoor settings are a setup for inevitable spread throughout the nursing home: "In such well-mixed spaces, one is no safer from airborne pathogens at 60 feet than 6 feet."

This model can well explain how the virus spread from our health worker and how our administrator contracted it despite both having worn appropriate protective gear and neither having come into physical contact with either residents or other health workers: once the virus was present in the nursing home, apart from spreading to the residents' rooms, 
small aerosol droplets also contaminated the nursing station and the administrator's office, which is situated near some of the residents' quarters. While small aerosol droplets disseminate more slowly than large ones, if an individual remains in a contaminated indoor region long enough, this secondary spread mechanism may well come into play. Moreover, while our workers were always masked, the residents were not, further facilitating transmission to both staff members and other residents. To describe the mechanics of spread, I propose an analogy with which most of us are familiar the use of a portable convector heater: its immediate and most potent effect is to warm the area to which its fans direct the heat. This may be compared to a large droplet that emits short-range contagion. However, if left on long enough, the convected heat will spread throughout the entire room and beyond, and under certain circumstances people some distance away from the heater will feel the warmth it provides. This is analogous to the mechanism of small droplet spread, which, while lower, is not always negligible.

2. A second factor is that some of the spread begins at a pre-symptomatic stage, when no one is aware that the virus is present. There is a difference between (lighter) precautionary behavior protocols that are adopted in order to "stay on the safe side" and the heavier measures taken when viral presence is known. Xi He et al., ${ }^{14}$ in discussing the significance of the pre-symptomatic stage, declared, "Our analysis suggests that viral shedding may begin 5 to 6 days before the appearance of the first symptoms." One frustrating conclusion they reach is the following: "We estimated that 44\% (95\% confidence interval, $30-57 \%$ ) of secondary cases were infected during the index cases' presymptomatic stage." Furthermore, they have reached the sobering conclusion that "Significant pre-symptomatic transmission would probably reduce the effectiveness of control measures that are initiated by symptom onset, such as isolation, contact tracing and enhanced hygiene or use of face masks for symptomatic persons." The stable door may well have been closed only after the horse had bolted. In short, they assert that much of the viral transmission occurs before the administration has any idea that the virus is present; and when that is the case, reactive strict adherence to COVID-19 protective protocols after the fact may not be totally effective.

3. A third explanation that makes sense intuitively, but for which there is only indirect support, relates to viral load. That is, that the greater the viral load, the more the virus will be liable to spread. Little et al. ${ }^{16}$ report that experiences with Middle East respiratory syndrome (MERS) - another coronavirus - and with household members who treat COVID-19 patients, together with research on animal models, all suggest that the higher the viral load, the more contagious the virus becomes. It would be reasonable to assume that in an enclosed nursing home containing, at times, six COVID-positive residents, the viral load was substantial.

One should not conclude from this analysis that COVID-19 precautions are ineffective; just that, under certain circumstances, such as a high community viral incidence and an enclosed indoor nursing home environment with shared rooms, even strict precautions will not always be completely effective.

I should like to presume that the precautions we implemented did play a role in somewhat reducing the nursing home's viral load, as most of our cases were mild and deaths were few.

\section{After the Fact}

The identification of a COVID-19-positive resident was a dangerous inflection point for the entire nursing home, as it heralded the onset of a crisis of a magnitude never previously faced. Nursing home function subsequently underwent a dramatic transformation.

Under ministry of health policy, all COVIDpositive residents had to be transferred out. A ministry of health affiliate removed asymptomatic residents to designated nursing homes equipped with COVID wards and decided who went where. Those who developed symptoms such as fever, cough or decreased oxygen saturation were transferred to hospital. However, this ostensibly straightforward algorithm - symptomatic patients are sent to hospital while asymptomatic residents are dispatched to designated COVID nursing home wards - is not always clear cut in reality. Two examples: If a resident had a high fever yesterday but is fine today, is he/she deemed symptomatic and therefore referred to hospital - or not? Another example: If the ministry has decided to refer a resident to nursing home "A," but the family prefers nursing home "B," should the family be accommodated when possible? Such instances were dealt with on an individual case-by-case basis.

\section{The Changes Implemented}

Firstly, until they were transferred out, residents who had tested positive needed to be completely separated from those who had not. Unfortunately, the transportation team itself was overwhelmed by parallel outbreaks elsewhere and had become so backlogged that we waited for an unwelcome day and a half before it arrived. Despite all our immediate isolation precautions and ministry support, these two delays, in test results and in transfer, offered the virus further opportunity to incubate and continue spreading within the nursing home. 
Secondly, all nursing home workers were immediately required to don special cumbersome and uncomfortable COVID-19 disposable overalls of the kind worn on COVID wards. These measures were improvisations, as our nursing home is not authorized to deal with COVID-19 patients on a regular basis.

The fact that all six positive residents were largely asymptomatic was encouraging, but we were nonetheless required to transfer them all out.

Thirdly, we went into lockdown protocol. Only essential workers continued to operate within the home; our multidisciplinary team, including myself, was locked out.

Our leadership triumvirate found itself with innumerable tasks at hand. Our administrator was responsible for overseeing the transfer out of COVID-19positive residents, which took place on a weekend, as it could be postponed no longer. That same day, another designated ministry team arrived to vaccinate our remaining residents and health workers; despite the outbreak, we were determined to Pfizer-vaccinate our residents at the first possible opportunity. Finally, as part of an ongoing process since the outbreak, we had ordered repeat testing to identify possible additional cases among the residents. In sum, three major events converged on a single day that weekend: group testing for COVID, vaccinations, and the transfer out of COVIDpositive residents.

Our head nurse began making room changes to separate COVID-positive from COVID-negative residents, leaving many alone in their rooms. While minimizing viral spread, this had the unfortunate effect of isolating them still further. The head nurse also had to ensure that our health workers knew exactly how to wear the awkward new COVID-19 protective gear and that they were following the latest stricter hygienic protocols used in the presence of the virus. At the same time, it was vital that she show understanding and support for the nurses and our health aides as their routine tasks became more burdensome. They were also legitimately fearful that they, too, might become infected with COVID.

As physician and medical director, I had the unenviable task of phoning the families of the COVID19-positive residents one after another, delivering the bad news and commiserating with them over their angst and the further isolation that they and their loved ones would yet have to endure, together with the danger that lay ahead. I know all these family members well, some of them for several years now, and their trust and grasp of the circumstances facilitated my task. However, I was not misled by their lack of outrage. I fully appreciated that the experience they were undergoing was terrifying for them.

At the same time, we also prepared an announcement to send out to our family WhatsApp group. This had to be formulated in a manner suitable for both categories of families: those related to a resident who had tested COVID-positive and those related to one who had tested negative. It needed to provide enough information to keep the families abreast of what was going on, without breaching confidentiality or being unduly technical. Furthermore, it needed to be sent out quickly, before false rumors could start spreading.

\section{Maintaining Contact with Families and Recipient Nursing Homes and Hospitals}

Once all that had been done, and the residents had been transferred, the situation in the nursing home itself calmed down. Nonetheless, because they were all our residents, we began contacting the various recipient institutions to receive daily professional updates on the status of those who had been transferred. In addition, of course, each family made its own inquiries. Success in communication with these institutions depended in part on the other party's availability and willingness to cooperate. In cases such as hospitals that were unwilling to provide telephone information to the referring physician for reasons of confidentiality, or institutions that did not regularly answer their phones, we worked in cahoots with a family member: if they received an update, they would inform us, and if we managed to get news, we would inform them. Even though I was not physically present in the nursing home during the outbreak, my days, which lasted far beyond nine to five, were spent dealing intensely with outbreakrelated tasks, the most poignant being fielding emotionladen telephone calls from family members who were anxiously tracking the progress of a hospitalized resident in unstable condition. They would ask me about the significance of a finding, what questions they should ask the treatment team, and how they should respond to scenario queries regarding insertion of nasogastric tubes, respiratory support, and intubation. It was inspiring to observe them all, one after another, trying to grasp the situation, take responsibility, and perform effectively under the most challenging conditions.

Regulations for family visits to the different locations varied. No one permitted a "regular" visit. Some allowed relatively close proximity, but only if the visitor were enveloped head to toe in COVID protection gear. Others offered viewing through a window. As anyone who has had a close relative hospitalized for COVID-19 knows only too well, this can be a frustrating and Kafkaesque experience.

For the first few days, we were concerned mainly with whether or not our infected residents would develop dangerous symptoms associated with COVID deterioration. Thankfully, in most instances, this did not happen. An encouraging factor was the understanding that, after several days, the longer these residents remained symptom-free, the less likely they were to develop COVID complications. When eventually deemed 
free of the disease, they would be permitted to return to our nursing home.

As COVID concerns declined, however, everyday nursing issues such as patients' confusion, agitation and constipation surfaced and worsened. In many cases, a stubborn problem that had grudgingly yielded to control in our nursing home reemerged as some of this control was lost in the transfer out. Moreover, the established mechanism that enabled a family member to speak with one of our nursing home workers, who knew their parent well, no longer existed. Nor did the recipient nursing home staff find it easy to deal with unfamiliar patients and family members. Furthermore, as the national crisis worsened, COVID wards in both the hospital and the specially designated nursing homes were on the verge of being overwhelmed.

While far less serious than an imminent COVID death threat, these ostensibly minor issues were nonetheless upsetting. After all, it was often problems such as these that had led the family to institutionalize their loved one in the first place. Worse still, some began as minor issues that later snowballed out of control. In one instance, a resident with a background of psychiatric illness, who was nonetheless medically stable, developed a new nursing problem in one of the recipient nursing homes. When her overall condition then worsened, she was referred to the hospital, where she was found to have non-COVID-related acute kidney failure and cardiac symptoms from which she had never suffered previously. We attributed her deterioration in part simply to her removal from a familiar institution to an unfamiliar one which, because of its preoccupation with containing the COVID crisis, gave less priority to nursing and other medical issues. In another instance, a diabetic patient with a minimal foot skin problem developed a significant distal diabetic ulcer within a matter of days. These disturbing incidents left family members and the rest of us speculating as to what could have / should have been done along the way to prevent them. They also reaffirmed a view I hold: I believe that nursing home residents, even those who are apparently stable medically, appear healthier than they actually are, because an effectively run nursing home provides a large measure of protection for these frail people. When this protective milieu is disrupted, their condition will not infrequently deteriorate.

After our administrator had herself recovered from the virus and was deemed non-contagious, she visited the two recipient nursing homes and met with our residents and the nursing home teams. There is no doubt that her visit improved overall communication with the recipient facilities, while also providing us with a clearer impression of the level of care our residents were receiving. Israel being a small country, we were not surprised to discover that we knew the administrator of one of the nursing homes and the medical director of the other.

Health ministry policy at the time deemed COVID-positive patients non-contagious 10 days after diagnosis, providing they had been asymptomatic for the previous 3 days and subsequently had 2 negative COVID results with at least a 24-hour interval between them. Those who didn't pass this test were released at day 20 , provided they had been symptom-free for the last 3 days. Finally, if between day 10 and 17 patients were well and had had two additional negative COVID results, they were returned to us after the second negative result was received. Slowly our residents began to come back. The extra work required to reintegrate them into our nursing home was a small price to pay for our profound sense of relief as we extricated ourselves from the outbreak, began to return to normal and were once more directly involved in their care. It also saved us from having to deal with the obstacles encountered in assessment of our residents while they were hospitalized in other institutions. We did everything we could to expedite their return as soon as possible, and, unsurprisingly, most of the acute nursing issues that had arisen while they were away dissipated within a few days of their return.

\section{Staff IIIness Issues}

Of the five symptomatic infected health workers, only one required hospital assessment. Thankfully, he improved, and was sent to convalesce at home. The administrative team members, including myself, maintained frequent contact with all our homebound workers and followed their progress until recovery, even though all had their own physicians. Our healthcare workers' salaries remained unaffected by COVID-19related interruptions, as Israel's social security department has a compensation system that reimburses staff for COVID-related absences.

At the same time, scheduling problems caused by their absence needed to be addressed promptly so that we could continue to provide orderly care for our residents. We were fortunate in that all our staff members were imbued with a sense of mission to get through the pre-outbreak and outbreak period as effectively as possible, and our workers displayed great scheduling flexibility. Some went beyond this. As an example, when there was a shortage of health aides, one of our registered nurses volunteered to do a few shifts as an aide.

We extended our shift length. While evidence has shown that there is greater likelihood of viral spread when workers are fatigued, there are also advantages to longer shifts. Indeed, even when there was no staffing shortage, we lengthened shifts to 2 in 24 hours, to reduce comings and goings within the nursing home. The CDC's only stipulated reservation is: "Avoid scheduling staff for more than 12 hours, if possible." 17 
We took care to express our appreciation for our teams' devotion regularly on WhatsApp, and our administrator, together with some of our locked-out multi-disciplinary team workers, brought along treats for the staff to enjoy during their shift. This "we are all in this morass together" atmosphere helped mitigate the stress our teams were enduring. As soon as the infected workers recovered from their illness and were deemed free of contagion, they returned to work.

\section{A Community Medical Issue Rather than Just a Patient Medical Issue}

By and large, physicians are trained to follow a particular patient with a particular problem. While this remains true of the situation described here, in this instance we are also dealing with a community-wide issue. I define "community" here as the aggregate of nursing home residents, their families, and the nursing home staff, including the administrative team. Analogous to a patient's having minimal symptoms initially, reaching a peak and then resolving, in this instance we had a nursing home health event (outbreak) in which several members of this community were initially infected, followed by a larger number that rose to a peak before subsiding. Apart from the two patients who died, all the others who had tested COVID-positive overcame the illness and returned to the nursing home and/or to work. Both professionally and emotionally, I found it convenient to treat the outbreak as a somewhat unified phenomenon in which the residents' illnesses were connected, rather than as a cluster of independent medical events.

\section{Physician Leadership Issues}

At its peak, our situation was particularly distressing: residents were dying, more and more were moved elsewhere, numerous staff members were at home with the virus, our COVID-positive administrator had been ill and was in isolation, and our nursing home was becoming depleted as every few days additional remaining residents were diagnosed as infected. These were undoubtedly difficult times.

Although I was only a salaried worker, and during the lockdown I was not physically present at all, I nonetheless felt an obligation to play a leadership role in guiding our administration, staff, and workers through the crisis. I found myself influential in providing succor for our administration team, the other workers, residents, and family members. I attribute my efficacy to the following factors:

- The physician as father figure: "The image of the father-figure has traditionally been assigned to the doctor and accepted by him."18 A reassuring message from the symbolic father figure of a physician has the potential to be especially effective. Even if, today, physicians take a less paternalistic approach and place greater emphasis on patient autonomy, I believe that, especially in times of crisis - and regardless of the physician's gender - the parental figure of the physician can unconsciously come into play and assuage fears.

- As terrible as COVID-19 is, in contrast to chronic ailments or irreversible events such as a CVA, for most of those affected it is an acute illness with a start and stop date. Physicians can use this knowledge and their experience of steering acute symptomatic illnesses to their resolution to assure those concerned that there is every reason to believe that things will get better.

- Now, many months into the pandemic, physicians are more familiar with the natural course of COVID. For example, we know that the longer a person remains asymptomatic after disease onset, the less likely he or she is to develop complications later, and that most of those afflicted, including the elderly, will get better. While many members of the public have Google and news access to this information, not everything they read is accurate or easy to interpret. Furthermore, I believe that, in times of crisis, information has greater impact when delivered by a familiar and authoritative figure such as the nursing home physician.

- As part of their training and experience, physicians become familiar with guiding sick people through their illnesses and bolstering them during the downturns. Supporting an individual or a community through a medical crisis requires similar emotional resources; however, the means of doing so has to be modified when dealing with an entire community rather than limiting provision of support to residents and their family members. I adopted a communityoriented approach both because all of us were dealing with the outbreak and I assumed that health workers and administrative staff who were part of the outbreak-affected community were also emotionally stressed and should be provided with appropriately tailored emotional support.

- Physicians are trained and experienced in delivering bad news. During the outbreak, we needed to address three different types of bad news: individual cases, including residents and health workers; parallel cases in which a number of patients simultaneously displayed overlapping symptoms; and disruption of the nursing home's normal functioning. Each category was dealt with slightly differently:

i. Families with an infected resident and health care workers were treated on a one-to-one basis.

ii. We used WhatsApp to convey group announcements and pass on relevant information to all the families simultaneously. The same approach, using the nursing home's WhatsApp worker group, was used to deliver information to staff members. 
iii. Finally, I provided support for our administrative team, which oversees the nursing home as a whole, assuring them that in due course our "stricken nursing home" would recover.

In updating our COVID routine since the outbreak, so far, other than one uninfected resident who has only received one vaccination and 2 COVID positive residents who remain unvaccinated, all health workers and remaining residents have received two Pfizer vaccinations. In accordance with ministry policy, we still do group COVID testing once weekly and will continue to do so for a while yet. Our workers still don masks, gloves, and gowns when necessary, and hand hygiene is still stressed.

These are some of the motifs and aspirations that guided us through this difficult period:

- Provision of good nursing and health aid care for our residents.

- Expressing ongoing appreciation and providing support for our front-line health workers.

- Maintaining continuous contact with families of residents who were transferred out.

- When possible, maintaining constant contact with the treatment teams in the institutions to which our residents had been temporarily transferred.

- Delivering bad news competently, compassionately, and honestly.

- Commiserating with families when a resident's condition deteriorated.

- Being available for families when death was approaching and inevitable.

Until the onset of the COVID pandemic, coping with multiple patients because of a single event was beyond the purview and experience of most physicians, to say the very least. Unless they work in a hospital E.R., by and large community physicians are accustomed to handling individual cases: while they may perhaps deal with several cases at once, they are unused to orchestrating an event in which a dozen or more patients are affected simultaneously. A nursing home outbreak confronted us with a new situation. I believe that, in addition to dealing with each case on its own merits, the physician has a responsibility to the community as a whole and can benefit from viewing such an outbreak not just as a collection of isolated events but rather as a group process that can be handled with much the same tools we use to cope with an individual case.

\section{SUMMARY}

As the nursing home physician, I found dealing with a COVID-19 nursing home outbreak a most difficult and challenging experience. It required me, as the physician, to be emotionally exposed and to accompany the suffering of an entire community - residents, their families, and the full complement of our health workers, each of whom had to deal with the innumerable obstacles and complications of his or her own personal predicament. Along the way there were casualties: some residents died; others, together with some health care workers, may suffer from residual COVID-19 complications; and some residents' nursing problems were exacerbated because priority was given to virusrelated interventions. Isolation angst was a common motif: residents were cut off from activities and from their families. Metaphorically, the situation was analogous to a war in which here and there battles were lost, but in which, overall, we managed to overcome the menace and return to our previous routine. I feel privileged to have witnessed firsthand so much personal heroism among residents who tolerated difficult conditions; among family members who sought to act in their loved one's best interest while at the same time grasping the complex reality the caregivers had to deal with; among the healthcare workers who overcame their fears and who strove to continue treating our residents with compassion and professionalism; and, finally, among members of our administration team, who oversaw events and assumed total responsibility while rendering an appalling situation more tolerable and pushing the wagon forward until we were able to return to our routine.

\section{References Références Referencias}

\footnotetext{
${ }^{1}$ https://www.pnas.org/content/118/1/e2015455118

2 https://www.sciencedirect.com/science/article/abs/pii/S0048
} 969720354759

3 https://agsjournals.onlinelibrary.wiley.com/doi/full/10.1111/ jgs. 16661

4 https://blogs.timesofisrael.com/\%e2\%80\%8fwhen-covid-19invaded-our-nursing-home/

5 https://www.nytimes.com/2020/03/21/us/coronavirusnursing-home-kirkland-life-care.html?searchResultPosition=1

6 https://agsjournals.onlinelibrary. wiley.com/doi/full/10.1111/ jgs. 16661

7 https://www.cdc.gov/coronavirus/2019-ncov/hcp/long-termcare.html

8 https://www.cdc.gov/coronavirus/2019-ncov/hcp/nursinghome-long-term-care.html

9 https://www.indeed.com/career-advice/career-development/ taking-a-sick-day

${ }_{10}$ https://agsjournals.onlinelibrary.wiley.com/doi/full/10.1111/ jgs. 16661

${ }_{11}$ https://agsjournals.onlinelibrary.wiley.com/doi/full/10.1111/ jgs. 16787

${ }_{12}$ https://www.who.int/publications/i/item/10665-336265

$13 \mathrm{https} / / / \mathrm{ww}$.medrxiv.org/content/10.1101/2020.08.26.2018 2824v2

${ }^{14} \mathrm{https}$ ://www.nature.com/articles/s41591-020-0869-5

${ }^{15} \mathrm{https}: / / \mathrm{www} \cdot \mathrm{bmj} . \mathrm{com} / \mathrm{content} / 369 / \mathrm{bmj} \cdot \mathrm{m} 1728$

${ }^{16} \mathrm{https}: / / \mathrm{www} . c d c . g o v / c o r o n a v i r u s / 2019-\mathrm{ncov} / \mathrm{hcp} /$ managingworkplace-fatigue.html

17 https://elischolar.library.yale.edu/cgi/viewcontent.cgi?article $=3109 \&$ context $=y m t d l$

$18 \mathrm{https} / / /$ elischolar.library.yale.edu/cgi/viewcontent.cgi?article $=3109 \&$ context $=y m t d l$ 